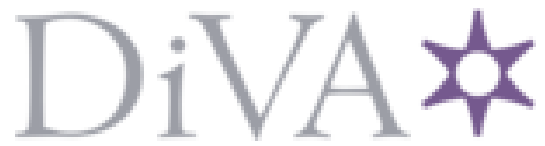

http://www.diva-portal.org

Preprint

This is the submitted version of a paper presented at ACM/SIGAPP Symposium on Applied Computing.

Citation for the original published paper:

Nešić, D. (2019)

Constructing Product-Line Safety Cases from Contract-Based Specifications

In: Proceedings of the Symposium on Applied Computing (pp. 2022-2031). Association for Computing Machinery (ACM)

N.B. When citing this work, cite the original published paper.

Permanent link to this version:

http://urn.kb.se/resolve?urn=urn:nbn:se:kth:diva-250230 


\section{Constructing Product-Line Safety Cases from Contract-Based Specifications}

\begin{abstract}
Safety cases are used to argue that safety-critical systems satisfy the properties determined to mitigate the potential hazards in the systems deployment environment. Although primarily a manual task, safety cases have been successfully created for single systems. However, when systems with a high number of configurations are considered, typically developed as a Product Line (PL), considering each possible configuration and constructing sound and complete safety-case argumentation is challenging. This paper presents a novel and general approach for the construction of a safety case for an arbitrary PL that is based on Contract-Based Specification (CBS) of the PL. Starting from a general CBS framework, a PL extension of the CBS framework is presented and it is shown that the extension preserves the properties of the original framework. Then, given a CBS specification of a PL, a set of transformation rules for the construction of the safety case argumentation-structure is defined. Finally, the approach is exemplified on a simplified, but real, and currently produced system by Scania CV AB.
\end{abstract}

\section{CCS CONCEPTS}

- Software and its engineering $\rightarrow$ Software product lines; Software safety;

\section{KEYWORDS}

Safety case, Product Line Engineering, Contract-based specification

\section{ACM Reference Format:}

1997. Constructing Product-Line Safety Cases from Contract-Based Specifications. In Proceedings of ACM Symposium on Applied Computing (SAC'19). ACM, New York, NY, USA, Article 4, 10 pages. https://doi.org/10.475/123_4

\section{INTRODUCTION}

Many software-intensive systems of systems are also safety critical. Indeed, failures of systems developed from domains like automotive, aerospace, or medical, can cause harm to material property or humans. Consequently, these domains are regulated and are required to comply with genral functional safety standards such as IEC 61508 [27] and with domain-specific standards such as ISO 26262 [28] in automotive, or DO 178C [34] in aerospace. These standards emphasize structured and traceable requirements engineering and require the creation of a safety case [3] to show compliance. A safety case is a structured argument, supported by a body of

Permission to make digital or hard copies of part or all of this work for personal or classroom use is granted without fee provided that copies are not made or distributed for profit or commercial advantage and that copies bear this notice and the full citation on the first page. Copyrights for third-party components of this work must be honored.

For all other uses, contact the owner/author(s)

SAC'19, April 2019, Limassol Cyprus

(C) 2016 Copyright held by the owner/author(s).

ACM ISBN 123-4567-24-567/08/06.

https://doi.org/10.475/123_4 evidence, showing that a system is designed and implemented to be acceptably safe in the environment in which it will be deployed.

As acknowledged in the literature [23, 29, 31], constructing and maintaining a safety case is a notoriously difficult task. First and foremost, safety cases are huge artifacts whose construction and maintenance is primarily manual [31]. For a reasonably sized system, just listing all the evidence such as safety analyses for different functional breakdown levels, requirements obtained from these analyses, test cases and test results verifying these requirements, results in a huge amount of information. Secondly, because different parts of the system are usually specified, designed, implemented, and verified using a variety of methods with different underlying semantics, it is especially difficult to create a sound argumentation structure which combines the available evidences into a safety case. Also, the lack of a unified semantics hinders automation and the creation of the argumentation structure is also most often manual.

Despite the difficulties, safety cases have been successfully developed in some domains, e.g. aerospace, but in other domains, e.g. automotive, they still represent a research challenge. The caveat separating these domains is the number of possible product configurations. A frequently used paradigm for designing and implementing a family of products is Product Line Engineering (PLE) [5, 30], where the commonalities and differences between product configurations are systematically managed, thus allowing a high level of reuse and quick creation of new product configurations. From the certification viewpoint, if the number of product configurations is low, like in aerospace domain, then it is feasible to create a safety case for each product configuration while reusing parts of safety argumentation between different safety cases. On the contrary, when the number of product configurations reaches hundreds of thousands [9], like in the automotive domain [42], this is not feasible both because it is impossible to produce the required evidence for each product configuration and because of the amount of manual work required to construct the argumentation structure for each safety case.

In this paper we propose a general and novel approach for the construction of a safety case for the complete Product Line (PL), instead of constructing a safety case per product configuration. The contribution of the paper is divided into two sub-contributions. The first sub-contribution is a PL extension of a general ContractBased Specification (CBS) framework $[6,8,11,33,40]$ which is then used to create a unified representation of the PL design and implementation. There are multiple reasons for relying on the CBS framework. Firstly, contemporary functional safety standards, for which PL safety cases are developed, emphasize structured and traceable requirements engineering which is one of the applications of CBS frameworks [8, 40]. Secondly, CBS frameworks are general enough to represent arbitrary systems and this removes the difficulty with the creation of a sound argumentation structure due to the need to consider artifacts based on different methods and languages. Thirdly, because CBS frameworks are compositional, i.e. the 
properties of all product configurations specified as requirements can be inferred from the properties of the constituent components, the need to analyze the properties of each system configuration in isolation is also avoided. Consequently the effort of creating the safety case is lowered. The second sub-contribution is the definition of a set of transformation rules from the CBS specification of a PL to a well-established safety-case notation, the Goal Structuring Notation (GSN) [2]. The result is, from a formal standpoint, a sound argumentation structure, and a set of verification obligations that if evidences showing that these obligations hold are provided, then the safety case complete-by-construction.

There are several notable approaches for safety case construction in the PLE context [14, 26, 35, 37]. Work in [26] presents a method for assurance of configurable architectures, based on established safety case patterns [15], and it enables explicit management of the argumentation belonging to each system configuration. Although the variability of the safety case argumentation is explicitly managed, the soundness and the completeness of the overall argumentation can be verified only by analyzing safety case argumentation for each system configuration. As discussed earlier, this is not feasible in domains with a high number of configurations and the approach in the present paper is purposely developed to avoid this issue. The approach in [14] is building on top results in [26] and automates the construction of a safety case for Simulink-based [12] specification of a PL. Although the automation reduces the effort of constructing a safety case for particular class of PLs, because of the underlying framework it is still necessary to perform perconfiguration analysis of safety case soundness and completeness. An approach based on safety contracts, can be found in [35, 37], and its implementation is part of the AMASS Tool Platform [4]. Although potentially scalable to PL safety cases, work in [35] and [37] primarily focuses on a concept from ISO 26262, the so-called Safety Element out of Context (SEooC). In contrast, the approach in the present paper considers safety cases for arbitrary PLs and leverages the CBS which is more formal and more expressive. Furthermore, unlike [35] and [37], the obtained safety case argues for the safety of the overall system in each of its configurations, compared to arguing only about the SEooC. A detailed discussion on related work, can be found in Section 6.

The paper is structured as follows. Section 2 summarizes the basics of PLE and CBS. Section 3 presents the CBS extension for specification of PLs. Section 4 defines the overall safety case argumentationstructure and defines the CBS to GSN transformation rules. Section 5 presents a real example which is modeled using CBS and for which a safety case is constructed. Section 6 discusses the related work while Section 7 concludes the paper.

\section{BACKGROUND}

This section summarizes the basic concepts of the PLE paradigm and CBS frameworks from existing literature.

\subsection{Product Line Engineering}

Product line engineering development paradigm [5, 30], facilitates the development of a family of systems that are jointly referred to as a product line. The central idea of PLE is to declare all functional and non-functional characteristics of each system in the PL, commonly referred to as features, and express these, together with any mutual dependencies, in a model where the most commonly used model is a feature model.

Definition 2.1 (Feature model). A feature model is a pair $\mathcal{V}=$ $(F, \mathfrak{C})$ where where $F=\left\{f_{1}, \ldots, f_{n}\right\}$ is a set of declared features which are either Boolean or Integer variables, and $\mathbb{C}$ is a set of Boolean constraints over the features in $F$.

An example of a constraint is $f_{1} \wedge f_{2}>100 \rightarrow f_{3}$. A value assignment to each of the features in $F$ is referred to as a product configuration.

Definition 2.2 (Product configuration). Given a feature model $\mathcal{V}$, a product configuration $\gamma$ is a set feature-value assignments $\gamma=\left\{f_{i}=\text { value }_{k}\right\}_{i=1}^{n}$. A product configuration for which each constraint in $\mathbb{C}$ evaluates to true is valid.

From hereon, terms product configuration and configuration will be used interchangeably and we will consider only valid configurations. For intuition, real industrial PLs often contain feature models with thousands of features and consequently define hundreds of thousands of valid configurations [9, 42].

Given a feature model, development artifacts are labeled with formulas expressed in terms of features and these formulas are known as presence conditions, denoted $\varphi$. They are written using the standard logical operators $\wedge, \vee, \neg$, arithmetic relations $>,<,=, \leq, \geq$, and their combinations. The purpose of presence conditions is to define the configurations to which a development artifact applies. By selecting a particular configuration, the artifacts that describe or implement the selected configuration are those whose presence conditions evaluate to true for the given feature value-assignment. In this way, a real-world product of a particular configuration can be derived automatically by selecting the features representing the characteristics of the product.

\subsection{Basic CBS framework}

In order to support rigorous design of complex and heterogeneous systems, several lines of research have presented CBS frameworks [6, $8,11,33,40,41]$. As noted in [7], one of the main strength of CBS is that it formally captures two central systems engineering principles: vertical design refinement across the development process, and horizontal composition of logical or physical components at a given abstraction level. Although with a slightly different notation, and with an emphasis on the use of CBS for requirements engineering, this section summarizes the CBS concepts presented in [39] and [8] that are relevant for safety case construction.

In order to illustrate the CBS concepts we use fragments from an application example (cf. Section 5) which is the Fuel Level Display (FLD) system that is a part of each Scania CV AB vehicle. The safety requirement on the FLD system is to ensure that the fuel volume indicated on the vehicle's instrumentation cluster, with some tolerances, corresponds to the actual fuel volume in the fuel tank. This is a safety requirement because indicating fuel volume higher that the actual one can lead to running out of fuel which in turn leads to sudden engine stop and loss of servo-steering which is essential for heavy vehicles due to heavy loads. 


\subsubsection{CBS concepts. Basic concepts of CBS are:}

i) component $C$,

ii) specification $S$,

iii) component implements a specification, denoted as $C \triangleright S$

iv) specification $S_{i}$ fulfills specification $S_{j}$, denoted full $\left(S_{i}, S_{j}\right)$,

v) n-ary component composition that results in new components, denoted as $C^{\prime}=C_{1} \otimes C_{2} \ldots \otimes C_{n}$.

A specification corresponds to a requirement and it specifies the intended behavior of a component which represents any logical or physical component. While here we refer to requirements as "specifications", other approaches refer to them as "assertions". The notion implements is similar across the majority of CBS frameworks and it corresponds to the expectation that the implementation of a component will exhibit the behavior specified in a specification. The notion of fulfillment between $S_{i}$ and $S_{j}$ represents the intention that if a component implements specification $S_{i}$ then it will also implement specification $S_{j}$. In [6], the term refinement is used to describe the same concept. Finally, composition correspond to the process of integrating existing components into new components.

Given the above concepts, we introduce the following definitions.

Definition 2.3 (Contract). A contract $K$ is an ordered pair of specifications, denoted $(A, G)$, where $A$ is called an assumption and $G$ is called a guarantee.

Definition 2.4 (Satisfy Contract). Component $C$ satisfies a con$\operatorname{tract}(A, G)$, denoted as $C \triangleright(A, G)$, if $\forall C_{e} \cdot C_{e} \triangleright A \Rightarrow C_{e} \otimes C \triangleright G$.

The main idea behind contracts is the separation of responsibilities. Given the contract $(A, G)$, the component $C$ can be developed independently of other components but when it is composed with a component $C_{e}$, often referred as the environment of $C$, which implements $A$, then the composition implements $G^{\prime}$. We consider that each contract is intended to be satisfied by one and only one component. This intention is expressed by saying that a contract $K_{i}$ is allocated to a component $C_{j}$, and denoted allTo $\left(K_{i}, C_{j}\right)$.

The intended semantics of the statement full $\left(S_{i}, S_{j}\right)$ is that the property expressed in $S_{i}$ logically entails the property expressed in $S_{j}$, denoted as $S_{i} \mid=S_{j}$. Given that specifications are implemented by components, the fulfills relation between specifications $S_{i}$ and $S_{j}$ expresses the intention that $\forall C . C \triangleright S_{i} \Rightarrow C \triangleright S_{j}$.

Assumption 1. The composition operator is commutative and associative.

The composition operator must be commutative and associative in order to enable that independently developed components can be composed in any order. We refer to components that are not composed of other components as atomic components and we refer to components composed of other components as composite components and say that a composite component has subcomponents. Atomic components usually represent low-level implementation like SW, HW, or mechanical components, but depending on the system's level of abstraction being considered, a SW module can be an atomic component while in other cases a single SW function can be an atomic component. On the other hand, composite components can represent everything from a high level system's functionality, down to a composition of two SW functions. From hereon, we introduce the shorthand $C^{\prime}=\bigotimes_{i=1}^{n} C_{i}$ that replaces $C^{\prime}=C_{1} \otimes \ldots \otimes C_{n}$ notation.

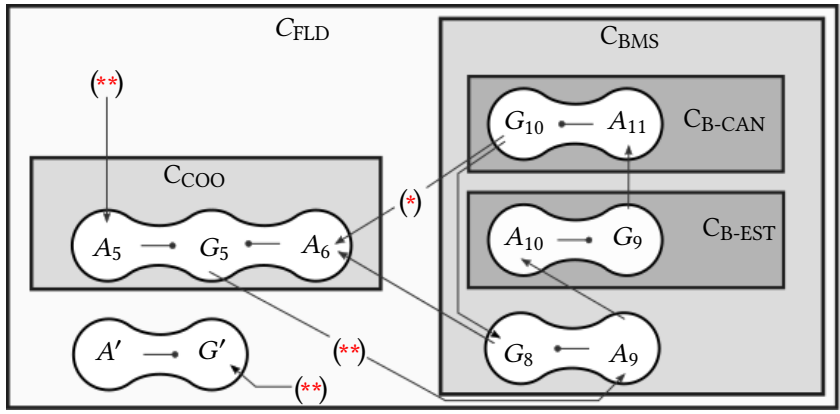

Figure 1: Graphical representation of CBS concepts.

Figure 1 shows the graphical representation of the CBS concepts introduced so far. Components are represented as rectangles and the composite component $C_{F L D}$ has subcomponents $C_{C O O}$ and $C_{B M S}$ that has further subcomponents $C_{B-E S T}$ and $C_{B-C A N}$. Contracts are depicted as white oval-like shapes and if a contract is overlaid over a component, that corresponds to the allocated to relation, e.g. allTo $\left(\left(A^{\prime}, G^{\prime}\right), C_{\mathrm{FLD}}\right)$. Each fulfills relation is represented by a line ending in an arrow, while the line ending with a small filled circle represents the assumption of relation which is intrinsic in each contract. Finally, the same specification can belong to multiple contracts, e.g. guarantee $G_{5}$ in contracts $\left(A_{5}, G_{5}\right)$ and $\left(A_{6}, G_{5}\right)$.

\subsection{Specification structure}

The assumptions and guarantees connected with fulfill and assumption $O f$ relations in Figure 1 form a graph that will be referred to as a specification structure. We will also say that a set of contracts and a set of components forms a specification structure.

Definition 2.5 (Specification structure). Let $C$ be a possibly empty set of components organized into a rooted tree, $\mathcal{K}$ be a possibly empty set of contracts such that each contract $\left(A_{i}, G_{i}\right) \in \mathcal{K}$ is allocated to a single component in $C_{i} \in C$, and the root component $C^{\prime} \in C$ is such that $C^{\prime}=\bigotimes_{j} C_{j}$ where $C_{j} \in C \backslash\left\{C^{\prime}\right\}$. Then, a specification structure $\mathfrak{D}$ for $C$ is a directed graph, i.e. $\mathbb{D}=(\mathcal{N}, \mathcal{E})$, if

i) each node $n \in \mathcal{N}$ is a specification $A_{i}$ or $G_{i}$,

ii) each edge $e \in \mathcal{E}$ corresponds to a single fulfill or assumption $O f$ relation between two specifications, and is denoted as $\left\langle S_{i}, S_{j}\right\rangle_{f}$ or $\left\langle S_{i}, S_{j}\right\rangle_{a}$,

iii) for each $\left\langle S_{i}, S_{j}\right\rangle_{a}$ and $\left\langle S_{i}, S_{j}\right\rangle_{f}$ it holds that $S_{i} \neq S_{j}$,

iv) for each edge $\left\langle S_{i}, S_{j}\right\rangle_{a}$ it holds that $S_{i}$ is an assumption, $S_{j}$ is a guarantee, and $S_{i}$ and $S_{j}$ is a contract $\left(S_{i}, S_{j}\right)$,

v) for each edge $\left\langle S_{i}, A_{j}\right\rangle_{f}$ it holds that

a) if $S_{i}$ is an assumption then it holds that if allTo( $\left.\left(S_{i}, G_{i}\right), C_{i}\right)$, and allTo $\left(\left(A_{j}, S_{j}\right), C_{j}\right)$ then $C_{j}$ is a subcomponent of $C_{i}$,

b) if $S_{i}$ is a guarantee then it holds that if allTo $\left(\left(A_{i}, S_{i}\right), C_{i}\right)$, and allTo $\left(\left(A_{j}, S_{j}\right), C_{j}\right)$, then there exists a component $C_{x}$ whose subcomponents are $C_{i}$ and $C_{j}$.

vi) for each edge $\left\langle S_{i}, G_{j}\right\rangle_{f}$ it holds that $S_{i}$ is a guarantee such that allTo $\left(\left(A_{i}, S_{i}\right), C_{i}\right), \operatorname{allTo}\left(\left(A_{j}, G_{j}\right), C_{j \neq i}\right)$, and $C_{i}$ is a subcomponent of $C_{j}$.

Def. 2.5 corresponds to the concept of contract structure in [40] and it provides an intuitive way to visualize how the property in the 
form of a guarantee $G^{\prime}$ allocated to the component representing the system $C^{\prime}$, is partitioned across the subcomponents of $C^{\prime}$. Moreover, the specification structure matches well with the ideas in functional safety standards, such as ISO 26262, which require the decomposition of higher level requirements into lower level requirements with explicitly managed traceability links. The example in Figure 1 is not a specification structure because relation $\mathrm{full}\left(G_{10}, A_{6}\right)$ violates condition (v)-b of Def. 2.5, i.e. $C_{C O O}$ and $C_{B-C A N}$ are not subcomponents of the same composite component. In a real system, this correspond to the scenario where the SW component $C_{B-E S T}$, a subcomponent of $C_{B M S}$, violates the architecture and bypasses the interface of $C_{B M S}$.

In order to ensure that satisfying the contract allocated to the system is a consequence of satisfying the contracts allocated to the atomic components, the following conditions is sufficient.

Definition 2.6 (Proper specification structure). A specification structure $\mathfrak{D}$ is proper if

i) for each assumption $A_{i}$ of a contract $K_{i}$ such that allTo $\left(K_{i}, C_{i}\right)$, there exists a specification $S_{k}$ and an edge $\left\langle S_{k}, A_{i}\right\rangle_{f}$, where $S_{k}$ is either an assumption such that allTo $\left(\left(S_{k}, G_{k}\right), C_{k \neq i}\right)$ and $C_{i}$ is a subcomponent of $C_{k}$ or $S_{k}$ is a guarantee such that allTo $\left(\left(A_{k}, S_{k}\right), C_{k \neq i}\right)$ and there exists a component $C_{x \neq k}$ whose subcomponents are $C_{i}$ and $C_{k}$,

ii) for each guarantee $G_{i}$ of a contract $K_{i}$ such that allTo $\left(K_{i}, C_{i}\right)$ where $C_{i}$ is a composite component $C_{i}=\bigotimes_{j} C_{j}$, there exits a guarantee $G_{j}$ and an edge $\left\langle G_{j}, G_{i}\right\rangle_{f}$, where allTo $\left(\left(A_{j}, G_{j}\right), C_{j}\right)$,

iii) $\mathfrak{D}$ is acyclic.

Going back to the example in Figure 1, even if the relation full $\left(G_{10}, A_{6}\right)$ would be removed, the example would still not be a proper specification structure because of all the relations labeled (**). Relation full $\left(G_{5}, A_{9}\right)$ introduces cycles, which violates condition (iii) of Def. 2.6, while the absence of fulfill relation to guarantees $A_{5}$ and $G^{\prime}$ violates conditions (i) and (ii), respectively.

The following theorem shows the key idea of CBS, i.e. the compositional reasoning about system properties and it corresponds to the dominance relation defined in [22].

THEOREM 2.7. Given a set of components $C_{i} \in C$, a set of contracts $K_{i} \in \mathcal{K}$ allocated to components from $C$ where $\mathcal{K}_{A} \subseteq \mathcal{K}$ is the set of contracts allocated to atomic components $C_{i}$, a contract $K^{\prime} \in \mathcal{K}$ such that allTo $\left(K^{\prime}, C^{\prime}\right)$ where $C^{\prime}$ is the root component, if

i) $\forall K_{i} \in \mathcal{K}_{A} \cdot \exists C_{i} \triangleright K_{i}$,

ii) contracts $K_{i} \in \mathcal{K}$ form a proper specification structure for $C$,

iii) $\forall \operatorname{full}\left(S_{i}, S_{j}\right) . S_{i}=S_{j}$,

then it holds that: $C^{\prime}-K^{\prime}$.

The theory that has been presented does not support specifying systems, i.e. composite components, that are configurable. In the next section, the presented theory is extended with PLE constructs.

\section{CBS EXTENSION FOR PLE}

This section presents the first part of the contribution, which is a PLE-oriented extension of the theory presented in Section 2.2 and Section 2.3. Before the formal definitions of the extension, we provide some intuition.
Consider using the CBS theory presented in the previous section for the development of a system with multiple configurations. This implies that each system configuration would have to be specified as a specification structure $\mathfrak{D}$, as indicated by the upper part of Figure 2, and the reasoning if each system configuration satisfies the allocated contract would be per-configuration. In order to avoid this, specification structures of individual system configurations can be merged into a common PL specification structure and then the reasoning about all system configurations can be performed simultaneously. The CBS model in the lower part of Figure 2 is a merge of the two configurations from the upper part of the figure where the dashed line of component $C_{B-E S T}$ indicates that this component exists only in some configurations of the $C_{F L D}$.

The applicability of a particular component in the merged model to a particular configuration is defined using presence conditions. As depicted by the bottom part of Figure 2, each component and each specification is labeled with a presence condition denoted $\varphi_{i}$. Contrary to the approach in [36] where presence conditions are assumptions of contracts, the presence conditions in the present paper are orthogonal to components and specifications because presence conditions of an artifacts and the artifact can evolve separately.

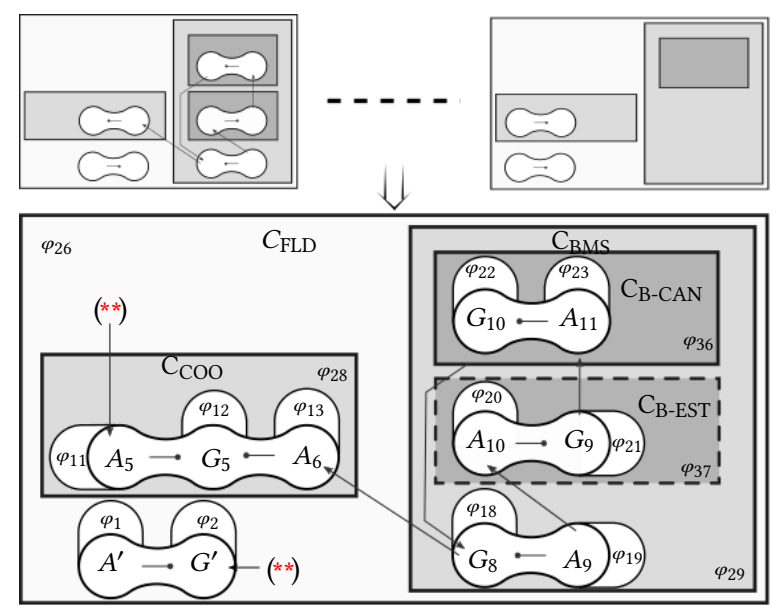

Figure 2: Example of a CBS model representing several configurations of the $C_{F L D}$ system.

In order to ensure that each configuration represented by the merged CBS model conforms to Def. 2.5 and Def. 2.6, different mismatches between presence conditions, and consequently between the corresponding artifacts, must be prevented. For example, in Figure 2, guarantee $G_{8}$ fulfills assumption $A_{6}$, and because component $C_{B M S}$ is intended to satisfy the contract $\left(A_{9}, G_{8}\right)$, presence conditions $\varphi_{18}, \varphi_{19}, \varphi_{29}$ and $\varphi_{13}$ must simultaneously evaluate to true or false for each configuration.

In the following two subsections, we formally define the PL specification structure and we define the constraints to which presence conditions must conform. 


\subsection{CBS model of a configurable system}

Assume that a feature model of a PL defines $n$ configurations $\gamma_{1}, \ldots, \gamma_{n}$. In order to avoid creating $n$ different specification structures, we introduce the PL specification structure $\widehat{D}$ which represents each of the $n$ configurations.

Definition 3.1 (PL Specification Structure). Let $C$ be a possibly empty set of components organized into a rooted tree, $\mathcal{K}$ be a possibly empty set of contracts such that each contract $\left(A_{i}, G_{i}\right) \in$ $\mathcal{K}$ is allocated to a single component in $C_{i} \in C$, and the root component $C^{\prime} \in C$ is such that $C^{\prime}=\bigotimes_{j} C_{j}$ where $C_{j} \in C \backslash\left\{C^{\prime}\right\}$. Then, a $P L$ specification structure $\widehat{\mathbb{D}}$ for $C$ is a directed graph $\widehat{D}=$ $(\mathcal{N}, \mathcal{E})$ such that

i) conditions (i)-(vi) of Def 2.5 hold,

ii) a labeling function $P$ labels each assumption $A_{i}$, guarantee $G_{i}$, and component $C_{i}$ with a presence condition $\varphi_{i}$.

Similarly to the basic CBS framework, we define a proper PL specification structure.

Definition 3.2 (Proper PL specification structure). A PL specification structure $\widehat{\mathfrak{D}}$ is proper if conditions (i) and (ii) of Def. 2.6 hold.

Because a proper PL specification structure is a superimposition of several configuration-specific specification structures, in the general case, it is possible that the graph of $\widehat{D}$ contains cycles and therefore the condition (iii) from Def. 2.6 does not apply to Def. 3.2. However, because allowing circular PL specification structures does not bring any conceptual benefits but increases the complexity of the following exposition, we introduce the following assumption.

Assumption 2. A PL specification structure $\widehat{\mathfrak{D}}$ is acyclic.

Before the constraints on presence conditions are defined, we introduce additional notation. $P(S)$ and $P(C)$ will be used to denote the presence condition of specification $S$ or a component $C$ while the notation $P_{\gamma}(S)$ will be used to denote the true or false value of the presence condition of $S$ or $C$ for a configuration $\gamma$. From here on, we consider that each presence condition evaluates to true or false for any given configuration $\gamma$.

\subsection{Constraints on presence conditions}

As outlined in section 2.1, artifacts are labeled with presence conditions in order to be able to automatically obtain the set of artifacts that apply to a certain configuration $\gamma$ by evaluating each presence condition and selecting the ones whose presence conditions evaluate to true.

We say that a PL specification structure $\widehat{\mathfrak{D}}$ is instantiated for a configuration $\gamma$, if each $A_{i}, G_{i}$, and $C_{i}$ whose presence condition evaluate to false for a given $\gamma$, is removed from $\widehat{\mathfrak{D}}$. The result of instantiation, denoted as $\mathfrak{D}_{\gamma}$ is a directed graph which is not necessarily a specification structure according to Def. 2.5 because of the previously described presence condition mismatches. In order to ensure that instantiation results in a specification structure, we introduce the following definitions and we refer to them as invariance with respect to configurations, hereinafter only invariant. In the following definitions, the symbol $\mid=\mathbb{C}$ represents logical entailment with respect to the set of constraint in the set $\mathfrak{C}$ of a feature model
$\mathcal{V}$. More formally, $f_{i}=\mathfrak{C} f_{j}$ is equivalent to $f_{i}, \mathfrak{C}=f_{j}$. For example, $f_{1} \wedge f_{2} \vDash f_{3}$ but if the set $\mathbb{C}$ contains the constraint $f_{1} \rightarrow f_{3}$ then it holds that $f_{1} \wedge f_{2}=\mathfrak{c} f_{3}$.

Definition 3.3 (Invariant Assumption). Given a specification $S$ and set of specification $S_{1}, \ldots, S_{n}$ where $S$ is the assumption of contracts $\left(S, S_{1}\right) \ldots\left(S, S_{n}\right)$, assumption $S$ is invariant if $P(S) \vDash=\mathbb{C}$ $\bigvee_{K=\left(S, S_{i}\right)} P\left(S_{i}\right)$.

Definition 3.4 (Invariant Guarantee). Given a specification $S$ and set of specification $S_{1}, \ldots, S_{n}$ where $S$ is the guarantee of contracts $\left(S_{1}, S\right) \ldots\left(S_{n}, S\right)$, guarantee $S$ is invariant if $P(S) \quad=_{\mathbb{C}}$ $\bigvee_{K=\left(S_{i}, S\right)} P\left(S_{i}\right)$.

Definition 3.5 (Invariant Allocation). An allocation of a contract $(A, G)$ to a component $C$ where $A$ and $G$ are an invariant assumption and guarantee, respectively, is invariant if $P(G) \models_{\mathbb{C}} P(C)$ and equally if $P(A) \mid=\mathbb{C} P(C)$.

Definition 3.6 (Invariant Composition). Composition of atomic components $C_{1}, \ldots, C_{n}$ into a composite component $C^{\prime}=\bigotimes_{i} C_{i}$ is invariant if it holds that $\forall C_{i} . P\left(C_{i}\right) \mid={ }_{\mathbb{C}} P\left(C^{\prime}\right)$.

Given a PL specification structure $\widehat{\mathbb{D}}$ with invariant assumptions, guarantees, allocation, and composition, instantiating $\widehat{\mathbb{D}}$ for an arbitrary configuration $\gamma$, results in a specification structure according to Def. 2.5.

Theorem 3.7. Given a set of configurations $\left\{\gamma_{i}\right\}_{i \in \mathbb{N}}$ and a PL specification structure $\widehat{\mathbb{D}}$, if

i) each assumptions is invariant,

ii) each guarantee is invariant,

iii) each allocation of a contract $\left(A_{i}, G_{i}\right)$ is invariant,

iv) component composition is invariant,

then each instantiation $\mathfrak{D}_{\gamma_{i}}$ is a specification structure.

Proof. The proof can be found in [1].

Theorem 3.7 established the conditions under which instantiating the PL specification structure $\widehat{\mathfrak{D}}$ for any configuration $\gamma$ will result in a specification structure. However, as shown by Theorem 2.7, proving that a property, in the form of a guarantee of a contract allocated to the system is satisfied, instantiating a PL specification structure must result in a proper specification structure.

Theorem 3.8. Given a set of configurations $\left\{\gamma_{i}\right\}_{i \in \mathbb{N}}$ and a PL specification structure $\widehat{\mathfrak{D}}$ such that each instantiation $\mathfrak{D}_{\gamma_{i}}$ is a specification structure, if

i) for each assumption $A_{i}$ of a contract $K_{i}$ where allTo $\left(K_{i}, C_{i}\right)$ it holds that $P\left(A_{i}\right) \mid=\left(\mathbb{C} \bigvee_{\text {full }}\left(S_{k}, A_{i}\right) P\left(S_{k}\right)\right.$ where $S_{k}$ is either $a$ guarantee of a contract $K_{k}$ such that all $\mathrm{To}\left(K_{k}, C_{k}\right)$ and exists a component $C_{x}$ whose subcomponents are $C_{i}$ and $C_{k}$, or $S_{k}$ is an assumption of a contract $K_{k}$ such that allTo $\left(K_{k}, C_{k}\right)$ and $C_{i}$ is a subcomponent of $C_{k}$,

ii) for each guarantee $G_{j}$ of a contract $K_{j}$ where allTo $\left(K_{j}, C_{j}\right)$ and $C_{j}$ is a composite component, it holds that $P\left(G_{j}\right) \quad=_{\mathbb{C}}$ $V_{\text {full }\left(G_{i}, G_{j}\right)} P\left(G_{i}\right)$ where each $G_{i}$ of a contract $K_{i}$ is such that allTo $\left(K_{i}, C_{i}\right)$ and $C_{i}$ is a subcomponent of $C_{j}$,

then each $\mathfrak{D}_{\gamma_{i}}$ is proper.

Proof. The proof can be found in [1]. 
A direct consequence of Theorem 3.7 and Theorem 3.8 in conjunction with Theorem 2.7 is the following corollary.

Corollary 3.9. Given a set of configurations $\left\{\gamma_{i}\right\}_{i \in \mathbb{N}}$, and a PL specification structure $\widehat{\mathbb{D}}$, if

i) premises of Theorem 3.7 hold,

ii) premises of Theorem 3.8 hold,

iii) $\forall \operatorname{full}\left(S_{i}, S_{j}\right) . S_{i}=S_{j}$

iv) for each contract $K_{i}$ allocated to an atomic component $C_{i}$ it holds that $C_{i}-K_{i}$,

then it holds that $C^{\prime} \triangleright\left(A^{\prime}, G^{\prime}\right)$ for each $\gamma_{i}$.

If a PL specification structure is considered to be the PL design, then Corollary 3.9 summarizes all conditions that the design must satisfy (i-iii), and the condition that the implementation must satisfy (iv) in order to be able to claim that each configuration satisfies the contract $\left(A^{\prime}, G^{\prime}\right)$. Given a particular PL specification structure, providing evidences that the premises of Corollary 3.9 hold, is the basis for construing a PL safety case in the next section.

\section{CONSTRUCTING A SAFETY CASE FROM $\widehat{D}$}

This section presents the second part of the contribution, which is a method for the construction of a safety case which is expressed using the GSN notation and which is based on a PL specification structure of a configurable system. Before presenting the safetycase construction method, the GSN notation is briefly introduced.

\subsection{Goal-Structuring Notation}

GSN notation [2] is one of the most prominent formalism for safety case representation. GSN is "a graphical argument notation which can be used to document explicitly the elements and structure of an argument and the argument's relationship to evidence" [2]. More specifically, GSN models form graphs called goal-structures. Figure 3 shows the GSN elements considered in the present paper. For a complete description of GSN notation, the reader is referred to [2].

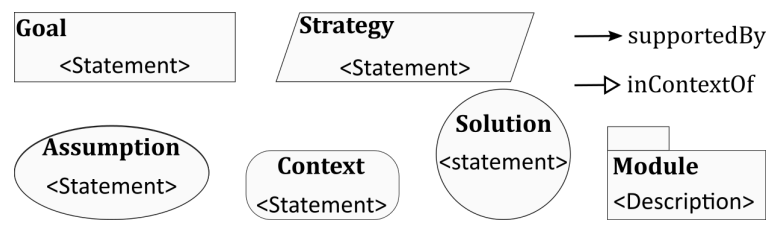

Figure 3: GSN elements used in the present paper

The elements in Figure 3 have the following meaning: i) Goal represents a claim about the system, ii) Solution represents the evidence expected to show that a goal has been met, iii) Strategy represents the rationale for decomposing a goal into sub-goals, iv) Assumption is the context in which a claim holds, v) Context explicitly declares the scope of a claim, and vi) Module is a container for self-contained piece of argumentation. The presented elements can be connected via the supportedBy link, which allows decomposing goals to subgoals and connecting them to solutions, or via inContext $O f$ links, which allow relating goals and evidence with assumptions and contexts.
In order to present a formal transformation of a CBS model to GSN notation, and in accordance with definitions from [15], a GSN model is defined as follows.

Definition 4.1 (GSN model). A GSN model $\mathcal{G}$ is a tuple, $\mathcal{G}=$ $\left(\mathcal{B}, \mathcal{A}, T_{\mathcal{B}}, T_{\mathcal{A}}, d\right)$, where:

i) $\mathcal{B}$ is a set of nodes, where each node is denoted as $b_{i}$

ii) $\mathcal{A} \subseteq \mathcal{B} \times \mathcal{B}$ is a set of edges, where each edge is denoted as $\left\langle b_{i}, b_{j}\right\rangle$,

iii) nodes and edges form a rooted, directed acyclic-graph,

iv) $T_{\mathcal{B}}$ is a labeling function $T_{\mathcal{B}}: \mathcal{B} \rightarrow\{$ Goal, Strategy, Solution, Assumption, Module\},

v) $T_{\mathcal{A}}$ is a labeling function $T_{\mathcal{A}}: \mathcal{A} \rightarrow\{$ supportedBy, inContext $O f\}$,

vi) $d$ is a labeling function $d: \mathcal{B} \rightarrow$ String which labels each node with a textual description,

vii) for each edge $T_{\mathcal{A}}\left(\left\langle b_{i}, b_{j}\right\rangle\right)=$ supportedBy it holds that $T_{\mathcal{B}}\left(b_{i}\right) \in$ $\{$ Goal,Strategy $\}$. If $T_{\mathcal{B}}\left(b_{i}\right)=$ Goal then $T_{\mathcal{B}}\left(b_{j}\right) \in\{$ Strateg $y$, Solution $\}$ and if $T_{\mathcal{B}}\left(b_{i}\right)=$ Strategy then $T_{\mathcal{B}}\left(b_{j}\right)=$ Goal,

viii) for each edge $T_{\mathcal{A}}\left(\left\langle b_{i}, b_{j}\right\rangle\right)=$ inContext $O f$ it holds that $T_{\mathcal{B}}\left(b_{i}\right)=$ Goal and $T_{\mathcal{B}}\left(b_{j}\right)=\{$ Assumption, Context $\}$.

\subsection{Constructing a safety case}

A safety case can only be developed with respect to a particular standard with which it should show compliance. In what follows, we consider the ISO 26262 functional safety standard for the automotive domain but the approach is general and it can be reused in other domains. Note that ISO 26262 contains nearly 800 requirements that are both process-based and product-based. Process-based requirements relate to the use of appropriate tools, the existence of appropriate expertise among the developers etc. This section focuses on product-based requirements.

In summary, product-based argumentation must show that the system requirements are sufficient to mitigate the potential hazards in the systems intended operating environment and that the system, in each of its configurations, implements these requirements. With this overall goal in mind, we propose the GSN model in Figure 4 as the overall argumentation-structure of the PL safety case.

The root node of the PL safety case is the claim that the "Product Line is safe", and the supporting argumentation is partitioned using the GSN modules in order to separate pieces of argumentation related to different systems. The argument that a particular system is safe is achieved by decomposing this claim into arguments about system safety with respect to each identified hazard, as exemplified in Module-System 1. One part of showing that a system is safe with respect to a certain hazard corresponds to arguing that overall system requirement, $G^{\prime}$ in Figure 4, mitigates the identified hazard. The evidence for this claim, as indicated by Figure 4, is in the majority of cases a manual review. As support for this review process, the GSN context nodes are used to refer to the artifacts that define the system and that have been produced while analyzing potential hazards based on which systems requirements were defined. On the contrary, the argumentation about the fact that the system implements the assigned property $G^{\prime}$ under the assumption $A^{\prime}$, can be fully developed from the PL specification structure of the system.

Arguing that the system satisfies the contract $\left(A^{\prime}, G^{\prime}\right)$ corresponds to arguing that the premises of Corollary 3.9 hold for the given system. In other words, besides the invariance conditions 


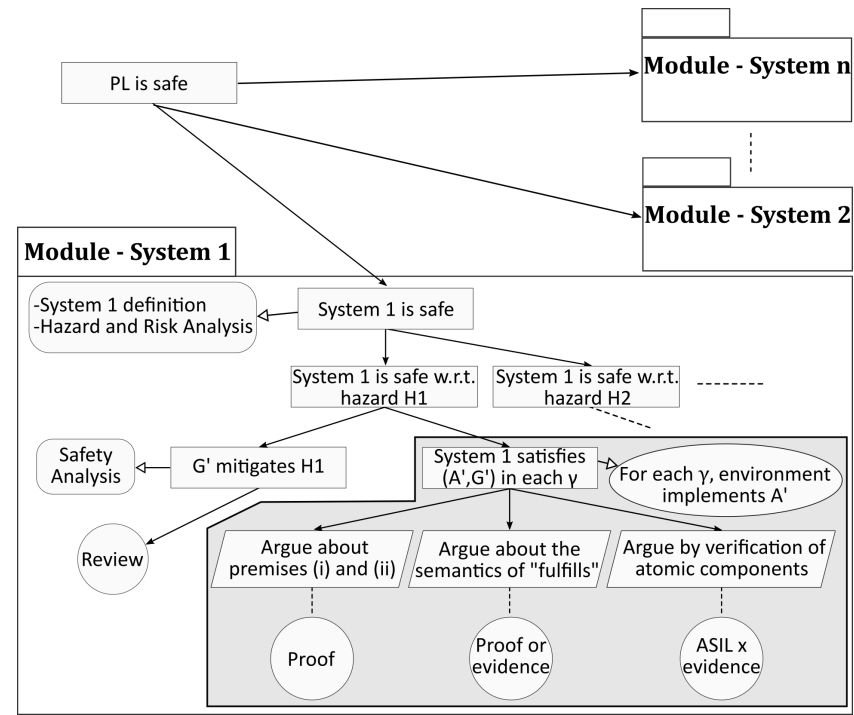

Figure 4: Overview of the PL safety case where the shaded part is constructed from the CBS specification of the PL.

on the PL specification structure, for each pair of specifications full $\left(S_{i}, S_{j}\right)$ it should be verified that $S_{i}=S_{j}$, and for each contractatomic component pair allTo $\left(\mathrm{K}_{\mathrm{i}}, \mathrm{C}_{\mathrm{i}}\right)$ it should be verified that $C_{i} \triangleright K_{i}$ by using SW or HW verification techniques such as testing, formal verification etc. Aggregating argumentation, supported by evidence, that all of the previously mentioned conditions hold, corresponds to an argument claiming that the system satisfies the contract $\left(A^{\prime}, G^{\prime}\right)$, where from a formal standpoint, and because it is based on a CBS model, this argument is complete-by-construction.

Before presenting the transformation rules from a PL specification structure to a GSN model, additional constraints must be enforced on a GSN model. For example, a single node $b_{i} \in \mathcal{B}$ is a valid GSN model but it does not represent a valid safety case.

Definition 4.2 (GSN safety case). A GSN model is a GSN safety case if

i) the root node $b$ is such that $T_{\mathcal{B}}(b)=$ Goal,

ii) for each node $b_{i}$ where $T_{\mathcal{B}}\left(b_{i}\right)=G o a l$, there exists a path to a node $b_{j}$ where $T_{\mathcal{B}}\left(b_{j}\right)=$ Solution.

The reason for introducing these conditions is that a safety case must argue that a certain claim holds, and each claim must be eventually supported by evidence.

\subsection{PL specification structure to GSN transformation rules}

Given an arbitrary PL specification structure $\widehat{\mathfrak{D}}$, the corresponding GSN safety case argumentation-structure is constructed as follows:

i) for each contract $\left(A^{\prime}, G^{\prime}\right)$ such that allTo $\left(\left(A^{\prime}, G^{\prime}\right), C^{\prime}\right)$ where $C^{\prime}$ is the composite component representing the system, create a GSN node $b_{i}$ such that $T_{\mathcal{B}}\left(b_{i}\right)=$ Goal and $d\left(b_{i}\right)=$ " $C^{\prime}$ satisfies $\left(A^{\prime}, G^{\prime}\right)$ for each configuration $\gamma^{\prime}$,

ii) create three GSN nodes $b_{1}, b_{2}, b_{3}$ such that $T_{\mathcal{B}}\left(b_{1}\right)=T_{\mathcal{B}}\left(b_{2}\right)=$ $T_{\mathcal{B}}\left(b_{3}\right)=$ Strateg $y$, and $T_{\mathcal{A}}\left(\left\langle b_{i}, b_{1}\right\rangle\right)=T_{\mathcal{A}}\left(\left\langle b_{i}, b_{2}\right\rangle\right)=$
$T_{\mathcal{A}}\left(\left\langle b_{i}, b_{3}\right\rangle\right)=$ supportedBy, and $d\left(b_{1}\right)=$ "Argue that premises (i) and (ii) of Corollary 1 hold", $d\left(b_{2}\right)=$ Argue that semantics of each fulfills relation holds", $d\left(b_{3}\right)=$ Argue by verification that contracts allocated to atomic components are satisfied".

iii) for each premise of Theorem 3.7 and Theorem 3.8 create a GSN node $b_{j}$ such that $T_{\mathcal{B}}\left(b_{j}\right)=$ Goal where $T_{\mathcal{A}}\left(\left\langle b_{1}, b_{j}\right\rangle\right)=$ supportedBy and $d\left(b_{j}\right)=$ "Premise $x$ holds" where $\mathrm{x}$ is one of the premises. Also, for each $S_{i}$ or $C_{j}$ that is in the scope of a premise $\mathrm{x}$, create a GSN node $b_{j s}$ where $T_{\mathcal{B}}\left(b_{j s}\right)=$ Solution, $T_{\mathcal{A}}\left(\left\langle b_{j}, b_{j s}\right\rangle\right)=$ supportedBy and $b_{j s}$ refers to the evidence about presence conditions entailment.

iv) for each pair full $\left(S_{i}, S_{j}\right)$ create a GSN node $b_{f}$ such that $T_{\mathcal{B}}\left(b_{f}\right)=$ Goal, where $T_{\mathcal{A}}\left(\left\langle b_{2}, b_{f}\right\rangle\right)=$ supportedBy and $d\left(b_{f}\right)=$ " $S_{i}$ entails $S_{j}$ ". Also, for each $b_{f}$ create a GSN node $b_{f s}$ where $T_{\mathcal{B}}\left(b_{f s}\right)=$ Solution, $T_{\mathcal{A}}\left(\left\langle b_{f}, b_{f s}\right\rangle\right)=$ supportedBy and $b_{f s}$ refers to the evidence of specification entailment.

v) for each pair allTo $\left(\left(A_{i}, G_{i}\right), C_{i}\right)$ where $C_{i}$ is an atomic component, create a GSN node $b_{v}$ such that $T_{\mathcal{B}}\left(b_{v}\right)=$ Goal, where $T_{\mathcal{A}}\left(\left\langle b_{3}, b_{v}\right\rangle\right)=$ supportedBy and $d\left(b_{v}\right)={ }^{\prime} C_{i}$ satisfies $\left(A_{i}, G_{i}\right)$. Also, for each $b_{v}$ create a GSN node $b_{v s}$ where $T_{\mathcal{B}}\left(b_{v s}\right)=$ Solution, $T_{\mathcal{A}}\left(\left\langle b_{v}, b_{v s}\right\rangle\right)=$ supportedBy and $b_{v s}$ refers to the evidence of contract $\left(A_{i}, G_{i}\right)$ being satisfied.

The presented transformation rules can be easily transformed into an algorithm that can be used to automate the creation of the safety case argumentation-structure. Given these transformation rules, and Def. 4.1 and Def. 4.2, next section presents an example application of the extended CBS framework and the transformation rules for construction of FLD system safety case.

\section{EXAMPLE APPLICATION}

Figure 5 depicts the PL specification structure for the subset of all configurations of the FLD system, and the corresponding CBSbased part of the safety case for the FLD system. This example is a simplification of the complete FLD technical architecture presented in [39], but unlike in [39], the example in Figure 5 considers several configurations of the FLD system in order to validate the PLE extension of the basic CBS framework.

The FLD system, represented by the component $C_{F L D}$, has subcomponents $C_{T A N K-A S S Y}$ representing the fuel tank assembly, $C_{C O O}$ representing the COOrdinator Electronic Control Unit (ECU), and $C_{B M S}$ representing the Body Management System ECU. Component $C_{T A N K-A S S Y}$ has further subcomponents $C_{T A N K}$ representing the actual fuel tank and two fuel level sensors; an ana$\log$ one represented by $C_{a-S E N S}$ and a digital one represented by $C_{d-S E N S}$. Component $C_{C O O}$ has subcomponents $C_{C-E S T}$ which is $\mathrm{C}$-function performing the fuel level estimation based on the sensor readings, and the subcomponent $C_{C-C A N}$ which is a C-function responsible for transmitting the estimated value over Controller Area Network (CAN) to the component $C_{I C L}$ which represents the vehicle Instrumentation CLuster (ICL). Similarly, the component $C_{B M S}$ has the subcomponent $C_{B-E S T}$ also performing the fuel level estimation but only in particular FLD configurations in which $C_{C O O}$ does not have this responsibility, and component $C_{B-C A N}$ which transmits the estimated value to $C_{C O O}$ which in turn passes it further to $C_{I C L}$. It should be noted that the FLD system architecture contains a single logical component representing the fuel 

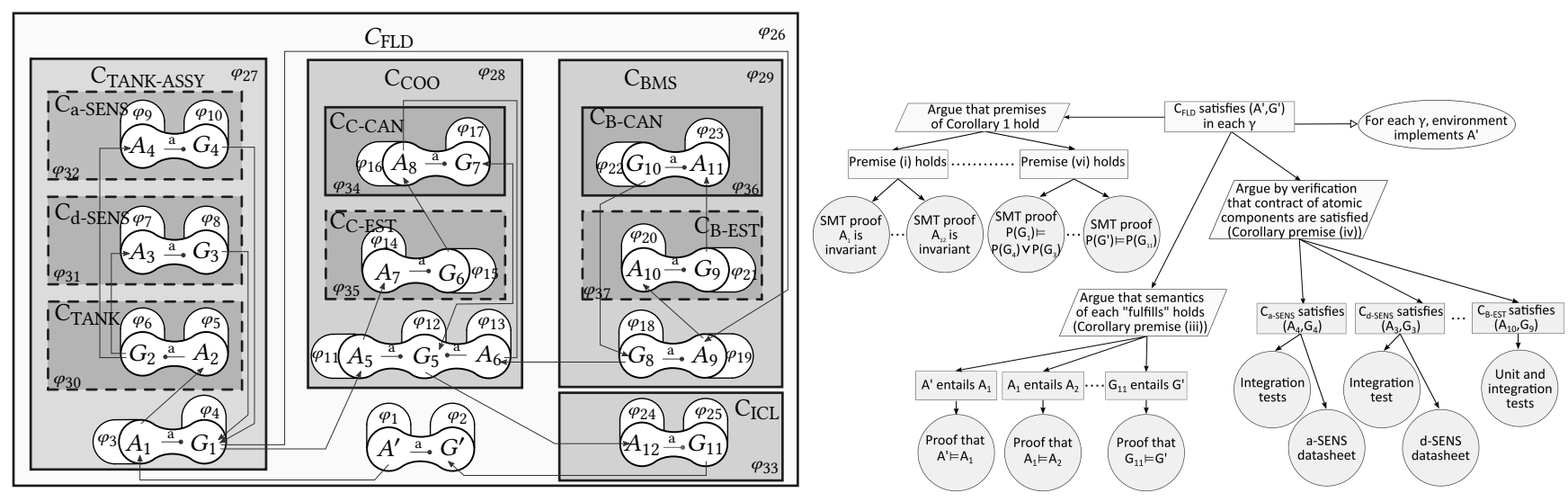

Figure 5: PL specification structure of the FLD system (left), and the corresponding safety case argumentation-structure (right)

volume estimator but because this component can be allocated to two different ECUs, components $C_{C-E S T}$ and $C_{B-E S T}$ capture the two different SW implementations of the same logical component.

Each component with a dashed border is used only in a subset of all $C_{F L D}$ configurations. Actually, each SW component exists in several production versions, each HW component exists in several variants, e.g. round or square $C_{T A N K}$, and each ECU exists in several variants depending on the ECU generation, e.g. generation 7 and 8 of $C_{C O O}$. Representing each of these is beyond the scope of the paper, and except $C_{a-S E N S}$ and $C_{d-S E N S}$ which explicitly represent two variants of the fuel volume sensor, all other components in Figure 5 represents a single version, variant, or a generation of a component. As described, the applicability of a particular component or a specification to a particular configuration is expressed through presence conditions ${ }^{1}$. The presence conditions $\varphi_{1}-\varphi_{33}$ were manually extracted from tools that manage requirements, SW and other artifacts. Due to space limitations, not all presence conditions are shown but for illustration purposes, several are presented in Table 1. Each term FX.Y represents one of more than 40000 Boolean features, e.g. F1.1 is the Truck feature while F1.2 is the Bus feature. $R D$ stands for release date which is a point in time when a certain component is released for production.

The contracts in the PL specification structure of the FLD, are based on the ones presented in [39]. However, because work in [39] considered only a single FLD configuration, the PL specification structure in Figure 5, contains additional contracts such as $\left(A_{6}, G_{5}\right)$ or all contracts allocated to $C_{\mathrm{BMS}}$ and its constituent components. These contracts were manually extracted from FLD system documentation by relying on principles presented in [39]. Besides the contract examples in Table 1, the PL specification structure of the FLD system contains contracts that guarantee that if the position of floater in $C_{T A N K}$ corresponds to the actual fuel volume, then either of the sensors can guarantee that the measured value corresponds to the actual fuel volume and consequently, the $C_{C-E S T}$ and $C_{B-E S T}$ can in different configurations guarantee that the estimated value will corresponds to the actual fuel volume and ultimately $C_{\mathrm{COO}}$

\footnotetext{
${ }^{1}$ Feature names have been modified due to confidentiality reasons.
}

Table 1: Examples of presence conditions and contracts

Presence conditions from the FLD system

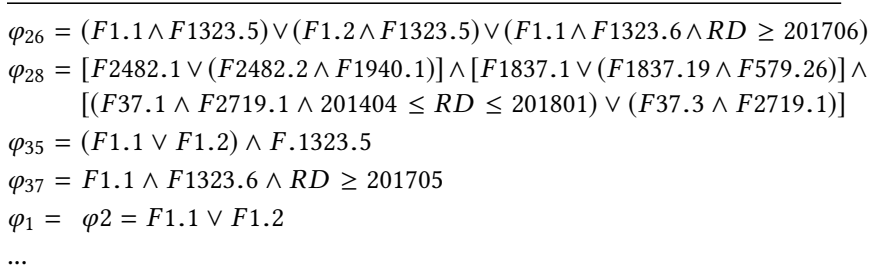

Contracts from the FLD system

$\mathrm{A}^{\prime}$ : $\quad$ The ignition state equals true.

$\mathrm{G}^{\prime}$ : Indicated fuel volume deviates at most $7 \%$ compared to actual fuel volume.

$\mathrm{A}_{12}$ : Estimated fuel volume value is updated every $1 \mathrm{~s}$.

$\mathrm{G}_{11}$ : Indicated fuel volume deviates at most $7 \%$ compared to actual fuel volume.

$\mathrm{A}_{5}$ : $\quad$ Measured fuel volume corresponds to the actual fuel volume.

$\mathrm{A}_{6}$ : Estimated fuel volume is received over CAN every $60 \mathrm{~ms}$.

$\mathrm{G}_{6}$ : Estimated fuel volume is transmitted over CAN every $90 \mathrm{~ms}$. $\cdots$

can transmit the estimated value over CAN to $C_{I C L}$ where the estimated value is indicated.

\subsection{Safety Case Argumentation-Structure}

Given the PL specification structure of the FLD system, the righthand side of Figure 5 depicts CBS-based part of the FLD safety case. According to the transformation rules from Section 4.3, the goal root node, arguing that " $C_{F L D}$ satisfies $\left(A^{\prime}, G^{\prime}\right)$ in each $\gamma$ ", is broken-down through three strategy nodes into sub-goals arguing that the premises of Corollary 3.9 hold.

While the construction of the GSN model on the right-hand side of Figure 5 is straightforward, judging if the evidence for each sub-goal can be obtained without disrupting enterprises processes and tools is the true test of the approach feasibility. The first set of evidences relates to proving that premises of Theorem 3.7 and Theorem 3.8 hold, i.e. proving logical entailment between Boolean 
expressions with respect to a set of Boolean constraints. Although the number of features is high, and the number of constraints in $\mathbb{C}$ is high, because the features and the constraints are primarily Boolean, encoding and checking the satisfiability of various entailments using efficient SMT solvers such as Z3 [13] is simple and feasible.

The second set of evidences relates to proving that for each pair of specification $\operatorname{full}\left(S_{i}, S_{j}\right)$ it holds that $S_{i}=S_{j}$. In the general case, this is a hard problem because specifications $S_{i}$ and $S_{j}$ can be such that it is not possible to formalize them and check entailment using a single technology. However, the observation from the analysis of the FLD system is that most often, as exemplified in Table 1, $S_{i}$ and $S_{j}$ are either identical as with $G_{11}$ and $G^{\prime}$ or even without formal analysis, it is obvious that $S_{j}$ fulfills $S_{i}$ as with $G_{6}$ and $A_{6}$. The reason for this is that when specifying a component in terms of a contract over component interface, not matching assumptions and guarantees of components is immediately counterintuitive. The third set of evidences relates to proving that for each contract and atomic component such that allTo $\left(K_{i}, C_{i}\right)$ it holds that $C_{i}$ satisfies $K_{i}$. Providing this type of evidences is the most difficult of the three because proving that $C_{i} \triangleright K_{i}$, means formally verifying the behavior of $C_{i}$. On the one hand, functional safety standards recommend that safety-critical software should be of low complexity and in addition formally verified. Here, formal verification approaches of SW against CBS specifications produce good results [24]. On the other hand, introducing large-scale formal verification into an enterprise as a part of everyday processes is still not realistic which means that majority of evidences for arguments of type $C_{i} \triangleright K_{i}$ will be based on testing as exemplified by the evidence discovered by analyzing the artifacts related to the atomic components of the $C_{F L D}$ system. Even without formal verification, verifying SW behavior using unit-testing is a de-facto standard in SW development practice and the creation of the third type of evidences is certain. The important thing to note is that by basing the PL safety case on the CBS framework which is compositional, the need to perform integration testing is removed and the already existing unit-testing process is sufficient for the production of evidences needed for the safety cases. Because testing is a technique that does not provide any guarantees, existing methods for the assessment of the confidence $[16,38]$ in safety case arguments can maybe be lifted to assessing confidence in a PL safety case.

From the safety case construction-process viewpoint, the presented method significantly reduces the amount of manual work because it defines a general argumentation structure applying to any PL of any size and complexity. Because each of the evidences is a precise verification obligation, detecting if the PL specification structure evolution leads to a violation of either of the obligations can be used for continuous analysis of the PL consistency and correctness. Furthermore, the approach allows iterative creation of the safety case because except the evidence needed to show that $C_{i} \triangleright K_{i}$, the premises of Corollary 3.9 can be verified stepwise in parallel with PL development. A relevant issue hindering complete automation of the presented approach is the need to access various types of information about the PL. Because such information is typically maintained by different tools, a lightweight data-integration [17] process that would merge and clean the necessary information is required. A promising approach for such data-integration are semantic web technologies as suggested in [32].

\section{RELATED WORK}

Two main approaches have been proposed for construction of safety case argumentation. The first line of research has focused on capturing safety case patterns that have been successfully used to argue for different properties $[10,15]$ while the second line of research has focused on generating safety case arguments from various types of artifacts [14, 26, 37]. In the area of PLE, most notable contributions $[14,18,19,21,26,36,37]$ come from the latter group.

Work in [25] uses safety-analysis artifacts to construct a safety case in the PL context which shows that contributions of identified faults to potential failures is acceptable. Work in [26] applies the method from [25] for creating a safety case in a PL context which argues that a configurable PL architecture is acceptably safe. However, unlike our approach, the approach in [25] does not consider the requirements that are defined for each identified fault and whether the system actually implements these requirements. The tool-supported method presented in [14] provides an automated way to construct a modular safety case based on the meta-models presented in [25] given input information from a variability management tool, architecture specification tool, and a hazard and safety-analysis tool. Work in [37] provides an approach for the creation of reusable safety cases fragments based on system specification in terms of weak and strong contracts that incorporate assume-guarantee reasoning. The motivation for the approach is based on the concept of Safety Element out of Context from the standard ISO 26262 and the essence of the approach is to perform hazard analysis and risk assessment using a variant of Fault Propagation and Transformation Calculus (FPTC) [20] and then map the output of FPTC onto introduced safety contracts. As argued in Section 1, the present paper considers development and assurance of arbitrary PLs and consequently relies on CBS paradigm where the constructed assurance case argues for system level properties instead of only SEooC. The line of research in $[18,19,21]$ introduces approaches for constructing safety case fragments that target particular requirements from various domain-specific safety standards. A notable aspect of these approaches is the attention to processes and their assurance with respect to standardized practices. Unlike this work, our aim is a general-purpose framework which enables constructing safety cases for arbitrary specifications allocated to configurations of a PL in the form of contracts.

\section{CONCLUSION}

As the criticality of the tasks performed by the systems from domains such as aerospace or automotive increases, the ability to construct safety cases for these systems is of outmost importance. Unfortunately, current methods for safety case construction fall short in scenarios when it is required to construct a safety case for a system with a huge number of configurations, i.e. for a PL.

In order to alleviate this problem, the present paper introduced a general and novel approach for the construction of a safety case for the complete PL, instead for each product configuration individually. In order to establish a unified representation of a PL, the first part of the contribution is an extension of a general CBS framework with the ability to specify a PL by using a mix of established CBS and PLE principles. The result of this extension is Corollary 3.9, which summarizes the constraints on CBS-based specification of PLs that 
preserve the compositional reasoning of the basic CBS framework. This allows reasoning about the properties of all PL configurations by inspecting only their constituent components. The second part of the contribution defines a set of transformation rules from a CBS-based specification of a PL to a PL safety case argumentationstructure expressed using the GSN notation. Because of the formal CBS foundations, the PL safety case argumentation-structure is sound and complete-by-construction. Moreover, the approach facilitates iterative safety case construction in parallel with the PL development, and a way to detect errors in the CBS model of a PL that lead to the inability to construct a PL safety case using the provided transformation rules.

Future work includes generalizations of the approach, and development of tool support that will enable large-scale case studies.

\section{ACKNOWLEDGMENT}

- and - are funded by the ECSEL PRYSTINE project and supported by Scania CV AB. - is funded by EU and VINNOVA via the ECSEL Joint Undertaking, agreement No. 692474, AMASS project.

\section{REFERENCES}

[1] [n. d.]. Theorem 3.7 and Theorem 3.8 proofs. Separate document created in order to preserve the double-blind process. https://www.dropbox.com/sh/ w9xfoj1efep91uc/AADcLWUNxQlKkZGVu34qn24Na?dl=0

[2] 2018. GSN community standard version 2. standard.

[3] 00-56 1996. 00-56: Safety Management Requirements for Defence Systems. standard. UK Ministry of Defence.

[4] AMASS Project Consortium. 2018. AMASS Tool Platform. https://www. amass-ecsel.eu/content/amass-prototype-p1

[5] Sven Apel, Don Batory, Christian Kästner, and Gunter Saake. 2016. Feature oriented software product lines. Springer-Verlag, Berlin-Heidelberg, Germany.

[6] Albert Benveniste, Benoit Caillaud, Alberto Ferrari, Leonardo Mangeruca, Roberto Passerone, and Christos Sofronis. 2008. Multiple Viewpoint ContractBased Specification and Design. In Proceedings International Symposium on Formal Methods for Components and Objects. 200-225.

[7] Albert Benveniste, Benoît Caillaud, Dejan Nickovic, Roberto Passerone, JeanBaptiste Raclet, Philipp Reinkemeier, Alberto Sangiovanni-Vincentelli, Werner Damm, Tom Henzinger, and Kim Guldstrand Larsen. 2015. Contracts for Systems Design: Theory. Research Report RR-8759. Inria Rennes Bretagne Atlantique ; INRIA.

[8] Albert Benveniste, BenoÃőt Caillaud, Dejan Nickovic, Roberto Passerone, JeanBaptiste Raclet, Philipp Reinkemeier, Alberto Sangiovanni-Vincentelli, Werner Damm, Thomas A. Henzinger, and Kim G. Larsen. 2018. Contracts for System Design. Foundations and TrendsÂ̋ in Electronic Design Automation 12, 2-3 (2018), 124-400. https://doi.org/10.1561/1000000053

[9] Thorsten Berger, Ralf Rublack, Divya Nair, Joanne M. Atlee, Martin Becker, Krzysztof Czarnecki, and Andrzej Wasowski. 2013. A Survey of Variability Modeling in Industrial Practice. In Proceedings of the Seventh International Workshop on Variability Modelling of Software-intensive Systems (VaMoS '13). Article 7 , $7: 1-7: 8$ pages.

[10] R. Bloomfield and K. Netkachova. 2014. Building Blocks for Assurance Cases. In Proceedings IEEE International Symposium on Software Reliability Engineering Workshops '14. 186-191.

[11] A. Cimatti and S. Tonetta. 2012. A Property-Based Proof System for ContractBased Design. In Proceedings Euromicro Conference on Software Engineering and Advanced Applications '12. 21-28.

[12] Richard Colgren. 2006. Basic Matlab, Simulink And Stateflow. AIAA (American Institute of Aeronautics \& Ast.

[13] Leonardo De Moura and Nikolaj Bjørner. 2008. Z3: An efficient SMT solver. In International conference on Tools and Algorithms for the Construction and Analysis of Systems. Springer, 337-340.

[14] André L. de Oliveira, Rosana T. V. Braga, Paulo C. Masiero, Yiannis Papadopoulos, Ibrahim Habli, and Tim Kelly. 2015. Supporting the Automated Generation of Modular Product Line Safety Cases. In Proceedings International Conference on Dependability and Complex Systems '15. 319-330.

[15] Ewen Denney and Ganesh Pai. 2015. Safety Case Patterns: Theory and Applications. Technical Report NASA/TM-2015-218492. NASA.

[16] Ewen Denney, Ganesh Pai, and Ibrahim Habli. 2011. Towards measurement of confidence in safety cases. In Proceedings International Symposium on Empirical Software Engineering and Measurement '11. 380-383.
[17] AnHai Doan, Alon Halevy, and Zachary Ives. 2012. Principles of Data Integration. Morgan Kaufmann Publishers Inc., San Francisco, CA, USA.

[18] Barbara Gallina. 2015. Towards Enabling Reuse in the Context of Safety-critical Product Lines. In Proceedings International Workshop on Product LinE Approaches in Software Engineering '15. 15-18.

[19] Barbara Gallina, Antonio Gallucci, Kristina Lundqvist, and Mattias Nyberg. 2013. VROOM \& cC: a Method to Build Safety Cases for ISO 26262-compliant Product Lines. In Proceedings International Conference on Computer Safety, Reliability and Security: SASSUR Workshop '13.

[20] B. Gallina, M. A. Javed, F. U. Muram, and S. Punnekkat. 2012. A Model-Driven Dependability Analysis Method for Component-Based Architectures. In Proceedings Euromicro Conference on Software Engineering and Advanced Applications '12. 233-240.

[21] Barbara Gallina, Shaghayegh Kashiyarandi, Karlheinz Zugsbratl, and Arjan Geven. 2014. Enabling Cross-Domain Reuse of Tool Qualification Certification Artefacts. In Proceedings International Conference on Computer Safety, Reliability and Security '14. 255-266.

[22] Susanne Graf and Sophie Quinton. 2007. Contracts for BIP: Hierarchical Interaction Models for Compositional Verification. In Proceedings Forum for Fundamental Research on Theory, Models, and Application for distributed systems '07. 1-18.

[23] William S. Greenwell. 2006. A taxonomy of fallacies in system safety arguments. In Proceedings International System Safety Conference '06.

[24] Dilian Gurov, Christian Lidström, Mattias Nyberg, and Jonas Westman. 2017. Deductive Functional Verification of Safety-Critical Embedded C-Code: An Experience Report. In Proceedings Critical Systems: Formal Methods and Automated Verification Workshop '17. 3-18.

[25] Ibrahim Habli. 2009. Model-based assurance of safety-critical product lines. Ph.D. Dissertation. Department of Computer Science, University of York, York, UK.

[26] Ibrahim Habli and Tim Kelly. 2010. A Safety Case Approach to Assuring Configurable Architectures of Safety-Critical Product Lines. In Proceedings International Symposium on Architecting Critical Systems '10. 142-160.

[27] IEC 61508 2010. ISO 61508: Functional Safety. standard. The International Electrotechnical Commission.

[28] ISO 26262 2011. ISO 26262: Road vehicles - Functional safety. standard. International Organization for Standardization.

[29] Zarrin Langari and Tom Maibaum. 2013. Safety Cases: A Review of Challenges. In Proceedings International Workshop on Assurance Cases for Software-Intensive Systems '15. 1-6.

[30] Frank J. van der Linden, Klaus Schmid, and Eelco Rommes. 2007. Software Product Lines in Action: The Best Industrial Practice in Product Line Engineering. Springer-Verlag, Secaucus, NJ, USA.

[31] Sunil Nair, Jose Luis de la Vara, Mehrdad Sabetzadeh, and Lionel Briand. 2014. An extended systematic literature review on provision of evidence for safety certification. Information and Software Technology 56, 7 (2014), 689 - 717.

[32] Damir Nešić and Mattias Nyberg. 2017. Applying Multi Level Modeling to Data Integration in Product Line Engineering. In Proceedings MODELS Satelite events: International Workshop on Multi-Level Modeling '17. 235-242.

[33] S. Quinton and S. Graf. 2008. Contract-Based Verification of Hierarchical Systems of Components. In Proceedings IEEE International Conference on Software Engineering and Formal Methods '08. 377-381.

[34] RTCA/DO-178C 2011. Software Considerations in Airborne Systems and Equipment Certification. standard. Radio Technical Commission for Aeronautics.

[35] Irfan Sljivo, Barbara Gallina, Jan Carlson, and Hans Hansson. 2014. Generation of safety case argument-fragments from safety contracts. In Proceedings International Conference on Computer Safety, Reliability, and Security '14. 170-185.

[36] Irfan Sljivo, Barbara Gallina, Jan Carlson, and Hans Hansson. 2016. ConfigurationAware Contracts. In Proceedings International Conference on Computer Safety, Reliability, and Security '16. 43-54.

[37] Irfan Sljivo, Barbara Gallina, Jan Carlson, Hans Hansson, and Stefano Puri. 2017. A Method to Generate Reusable Safety Case Argument-fragments from Compositional Safety Analysis. Journal of Systems and Software 131, C (Sep. 2017), 570-590.

[38] Rui Wang, Jérémie Guiochet, Gilles Motet, and Walter Schön. 2016. D-S Theory for Argument Confidence Assessment. In Proceedings Internation Conference on Belief Functions '16. 190-200.

[39] Jonas Westman. 2014. Specifying and structuring requirements on cyber-physical systems using contracts. Technical Report urn:nbn:se:kth:diva-159599. KTH.

[40] Jonas Westman and Mattias Nyberg. 2018. Conditions of contracts for separating responsibilities in heterogeneous systems. Formal Methods in System Design 52, 2 (01 Apr 2018), 147-192.

[41] Jonas Westman and Mattias Nyberg. 2018. Preserving Contract Satisfiability under Non-Monotonic Composition. In Accepted at Forum for Fundamental Research on Theory, Models, and Application for distributed systems '18. Madrid, Spain.

[42] Len Wozniak and Paul Clements. 2015. How Automotive Engineering is Taking Product Line Engineering to the Extreme. In Proceedings of the 19th International Conference on Software Product Line (SPLC '15). ACM, 327-336. 